\title{
Sistema medidor de amoniaco en la avicultura (megato)
}

\section{Poultry ammonia measuring system (megato)}

\author{
Estefany María González King ${ }^{1 *}$, Ricardo Aarón Morales Sánchez ${ }^{1}$, Amir Filos ${ }^{1}$ \\ ${ }^{1}$ Licenciatura en Ingeniería Industrial Administrativa - Sede La Chorrera - Universidad del Istmo.
}

\begin{abstract}
Resumen El sistema de medición de gases contaminantes en la avicultura es un proyecto de innovación social, desarrollado en pequeñas granjas auto sostenibles y con unidades vulnerables. Este será capaz de alertar a la población cuando los animales están en peligro por gases contaminantes producidos como el amoniaco. Estos gases son capaces de contaminar la carne del animal hasta provocar su muerte, generando pérdidas económicas en la granja y poniendo en riesgo la salud, ya que la carne es para consumo humano en dichas comunidades. El proyecto consiste en una placa controladora de bajo costo, conectada con un circuito de cables y sensores de gas de amoniaco, la cual emitirá niveles, que, al detectar peligro, enviará una programación aplicada al controlador que emite una alerta para tomar medidas como cambiar las camas del ave o ventilar el lugar para que el animal no sufra. Con el sistema de medición vamos a evitar la trasmisión de enfermedades, estos riegos mortales del animal hacia la población o consumidor del producto, también bridarle una manera de automatizar e incluir nuevas tecnologías en sus pequeñas granjas a bajo costo.
\end{abstract}

Palabras clave Automatizar, arduino, innovación social, sostenibles, contaminación, controlador.

\begin{abstract}
The measurement system for polluting gases in poultry farming is a social innovation project, developed in small self-sustaining farms with vulnerable units. This will be able to alert the population when animals are endangered by pollutant gases produced such as ammonia. These gases can contaminate the meat of the animal until causing its death, generating economic losses in the farm and putting in risk the health, since the meat is for human consumption in these communities. The project consists of a low-cost controller board, connected to an ammonia gas cable and sensor circuit, which will emit levels, which, upon detecting danger, will send a schedule applied to the controller that issues an alert to take measures such as changing the bird's beds or ventilate the place so that the animal does not suffer. With the measurement system we will prevent the transmission of diseases, these mortal risks of the animal to the population or consumer of the product, also provide a way to automate and include new technologies in their small farms at low cost.
\end{abstract}

Keywords Automate, arduino, social innovation, sustainable, contamination, controller.

* Corresponding author: estefanygk95@gmail.com

\section{Introducción}

El amoniaco se encuentra en niveles relativamente altos en todos los estiércoles animales, puede existir en muchas formas químicas y moverse fácilmente en el medio ambiente en forma de gas en el aire, un compuesto disuelto en el suelo, en la superficie del agua o una combinación de ambas. El nitrógeno en forma de amoniaco (NH3) es de particular importancia debido a su potencial para crear olores y dañar la calidad del agua, la salud humana y animal, es asociado, especialmente a la industria avícola.

El amoniaco presente en las proteínas de los alimentos es transformado en otros compuestos nitrogenados como urea y amoniaco, este último cuando entra en contacto con el aire, es un gas incoloro de olor penetrante que se disuelve fácilmente en el agua y tiende a evaporar con rapidez, por eso su control en las producciones animales es tan difícil. Se origina de la descomposición del ácido úrico de las excretas de los animales y organismos en descomposición. Estudios al respecto afirman que concentraciones de amoniaco de 50 a $100 \mathrm{ppm}$ en las instalaciones pueden generar síntomas respiratorios e incluso solo con $25 \mathrm{ppm}$ los parámetros productivos empiezan a verse afectados.

El ser humano detecta el amoniaco cuando este alcanza una concentración de 25 ppm o más, mientras que la concentración máxima que puede soportar es de 100 ppm durante ocho horas. Sin embargo, las aves pueden presentar diversos problemas cuando resultan expuestas durante largos períodos de tiempo a niveles tan bajos como de $20 \mathrm{ppm}$. Los altos niveles de amoniaco son seguramente los responsables de la misma que, aún ocasionando una baja mortalidad, puede producir grandes pérdidas económicas. Entre los síntomas típicos de la queratoconjuntivitis se encuentran el amontonamiento de las 
aves en grupo, el frote de los ojos con las alas y es que estos tienden a cerrarse y a hacerse sensibles a la luz. La incidencia más alta de queratoconjuntivitis tiene lugar cuando la granja no estaba en buenas condiciones y la concentración de amoniaco era muy alta.

El objetivo de la mayoría de los productores es evitar desde un principio las altas concentraciones de amoniaco, o bien controlar las inflamaciones inducidas y minimizar los efectos en la salud y el desempeño productivo de las aves; en la actualidad estos son los mecanismos utilizados para mitigar.

El suministro de una dieta equilibrada y completa es de suma importancia. Los problemas debidos a la genética de alto rendimiento, la formulación del alimento y la medicación pueden conducir a la producción de estiércol húmedo, causando un aumento del amoníaco y la liberación de olor junto a una reducción del desempeño productivo de los pollos de engorde y la eficiencia alimenticia.

Es necesario optimizar la densidad poblacional de las aves para ayudar a limitar el exceso de humedad en la caseta, reduciendo así los procesos anaeróbicos. Si los niveles de amoníaco aumentan, es necesaria una mayor ventilación. No obstante, esto solo debe realizarse de acuerdo con el clima y la temperatura de la caseta, Deben realizarse ajustes de temperatura con relación al bienestar y el clima de la caseta. El mejoramiento de la digestibilidad de los nutrientes puede lograrse suplementando las dietas con aditivos.

Nuestros objetivos son establecer y crear un nuevo método y mecanismo para alertar a la comunidad de una problemática y poder brindarles un sistema automatizado.

Este sistema automatizado de control de calidad de aire es usado para la detección de contaminación en el medio ambiente, por lo general es implementado en circuitos de control como alarmas en las casas, sitios donde se desea prevenir altos niveles de contaminación a nivel aeróbico como industrias que manejan compuestos químicos que pueden ser nocivos también para la salud, especialmente en equipos controladores de calidad de aire en edificios/oficinas, sin necesidad de realizar una inversión mayor, ya que es un proyecto de bajo costo y se enfoca en comunidades de bajos recursos.

\section{Material y métodos}

El lugar de ejecución del proyecto es la República de Panamá, en comunidades vulnerables, pequeños productores, proyectos de granjas auto sostenibles, escuelas y comunidades que tengan implementado la avicultura para el consumo propio.

Se instala un sensor que se encarga de la detección de concentración de amoniaco en diversos porcentajes, el MQ-137 es dual, de carácter analógico y digital. La señal digital, presenta niveles por lo que puede ser procesada por un microcontrolador. Se utilizan en equipos de control de calidad del aire para edificios y oficinas, son adecuados para la detección de NH3. Este sensor es sensible en similar proporción a los gases mencionados, con lo que podemos determinar si el aire está limpio.

El controlador y sensores modelo mq137 se encuentran dentro del área de trabajo, para ejecutarlo en diferentes áreas de la galera.

El controlador es una placa electrónica, que cuenta con 14 entradas/salidas digitales, de las cuales seis se pueden utilizar como salidas PWM (Modulación por ancho de pulsos) y otras seis son entradas analógicas. Además, incluye un resonador cerámico de $16 \mathrm{MHz}$, un conector USB, un conector de alimentación, una cabecera ICSP y un botón de reseteado. La placa incluye todo lo necesario para que el controlador pueda ejecutar los valores que requiere una vez conectado a un ordenador con un cable USB o a la corriente eléctrica a través de un transformador.

\section{Especificaciones}

\section{[1] Model No.: MQ137}

Tipo de sensor: Semiconductor

Encapsulación estándar: Bakelite (Black Bakelite)

Gas: Ammonia

Concentración: 5-500ppm (Ammonia)

\section{Circuito}

Voltaje del bucle (Vc): $\leq 24 \mathrm{~V}$ DC

Voltaje del calentador ( $\mathrm{VH}): 5.0 \mathrm{~V} \pm 0.2 \mathrm{~V}$ AC or DC

Resistencia de la carga (RL): Ajustable

\section{Características del sensor}

Resistencia del calentador (RH): $31 \Omega \pm 3 \Omega$ temperatura ambiente

Consumo del calentador (PH): $\leq 900 \mathrm{~mW}$

\section{Gráficos, fotografías y tablas}

Consiste en brindar un mecanismo capaz de medir gases tóxicos en galeras de aves por medio de un programa diseñado bajo el mando de un controlador, las mediciones serían constantemente y alertaría inmediatamente a las comunidades que se encuentran involucradas específicamente en granjas auto sostenibles. Además de alertar, queremos educar a la población sobre los gases que se crean de la combinación de las heces fecales de las aves, otros residuos y productos utilizados en las granjas; estos gases producen la contaminación de la carne y muerte del animal. Este sistema es un proyecto de innovación social, donde los principales beneficiados son personas de comunidades vulnerables y granjas auto sostenibles. 


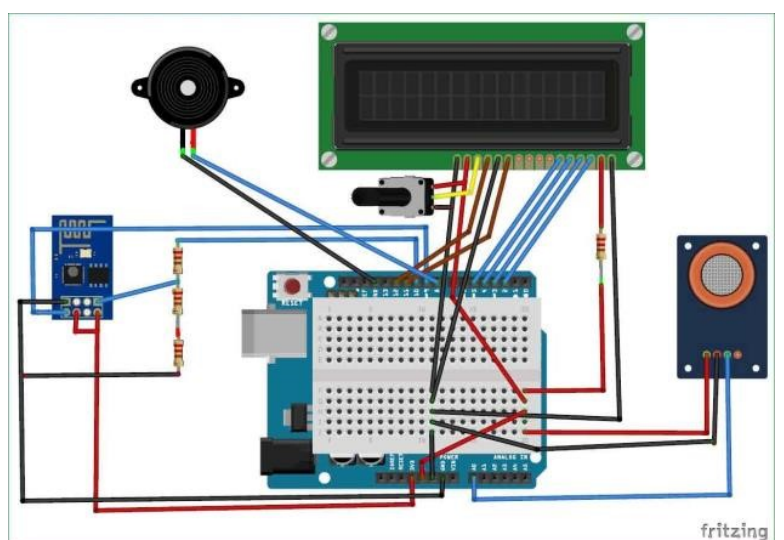

Figura 1. Conexión del circuito hacia el controlador Arduino.

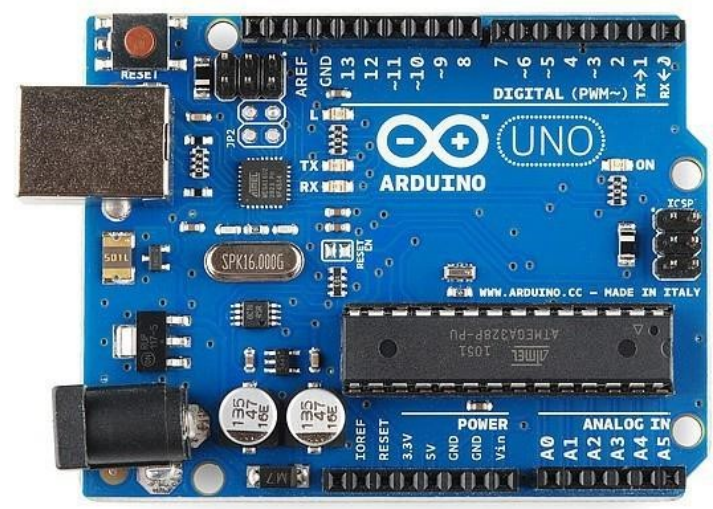

Figura 2. Controlador del sistema; Instrumento Arduino.

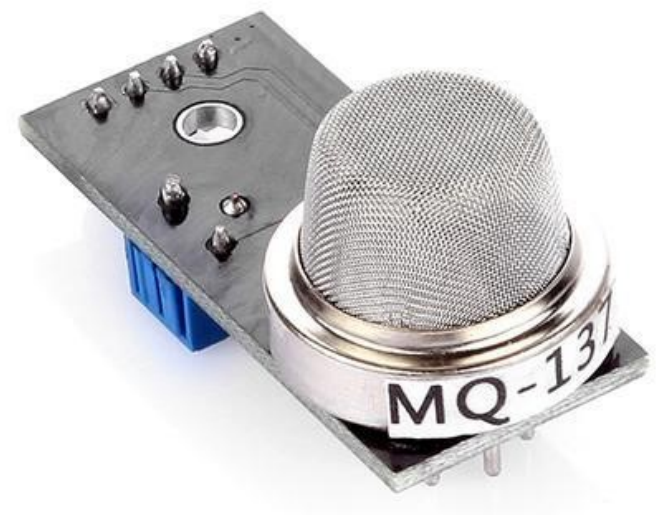

Figura 4. Sensor MQ137.

\section{RESULTADOS}

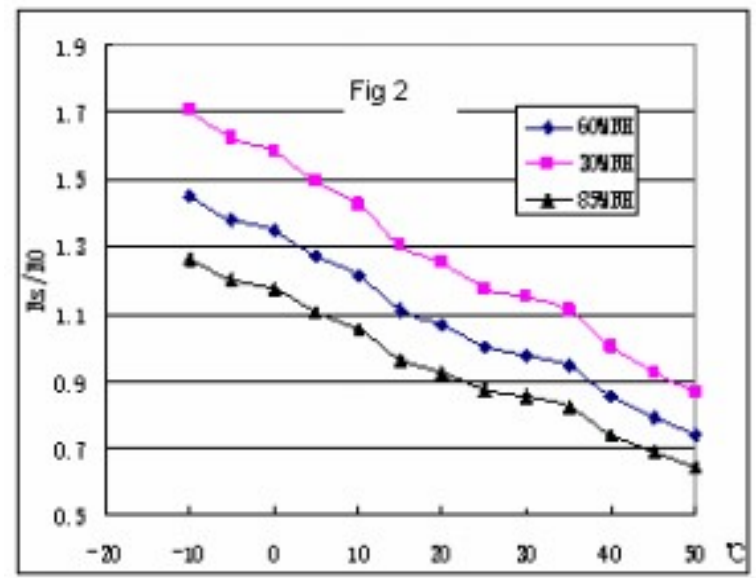

Figura 5. Influencia de la temperatura /humedad.

\section{Conclusiones}

Nuestro proyecto busca acercar las nuevas tecnologías a comunidades que las necesiten, lograr innovar socialmente el desarrollo tecnológico, automatizando su trabajo; queremos aplicarlos en áreas que resulten beneficioso y lograr su expansión, además dentro de nuestro proyecto tenemos mejoras continuas como aplicaciones de nuevos mecanismos para beneficiar a la comunidad, brindándole la facilidad de una galera automatizada a bajo costo, y disminuir el precio y elementos de calidad. El proyecto se podrá lograr con la ayuda de financiamiento de distintas áreas, al ser un proyecto atractivo para comunidades vulnerables y de responsabilidad social.

El elaborar este proyecto toma la capacidad de unir ciencias como la robótica, tecnología, programación, agricultura e innovación social. Esperamos alcanzar que las personas sean capaces de identificar un riesgo sin necesidad de invertir grandes sumas de dinero.

\section{REFERENCIAS}

[1] "MQ137 Semiconductor Sensor for Ammonia" Disponible en: https://goo.gl/xomvZ9

[2] "Página web Arduino" Disponible en: https://bit.ly/1ThOjzl

[3] "Página web Arduino" Disponible en https://bit.ly/1ThOjzl 